\title{
NEUROPHILINS AND THE NERVOUS SYSTEM
}

\author{
DANIEL D. MIKOL, MD, PhD, and EVA L. FELDMAN, MD, PhD
}

University of Michigan, Department of Neurology, 4414 Kresge III, 200 Zina Pitcher

Place, Ann Arbor, Michigan 48109, USA

Immunosuppressive drugs are being used increasingly to suppress rejection of organ transplants and to treat autoimmune disorders. Cyclosporin A (CsA), FK506, and rapamycin are among a class of immunosuppressants whose mechanisms of action are similar and which have unique neurological toxicity. Cyclosporin A and FK506 lack structural similarity yet have similar immunosuppressive effects, whereas rapamycin is structurally similar to FK506 but has a distinct spectrum of biological activities. ${ }^{6}$ These immunosuppressants are membrane-permeable prodrugs which mediate their effects after first binding to a variety of cytosolic proteins called "immunophilins" due to their affinity for immunosuppressant ligands. Intriguingly, concentrations of immunophilins are often greater in the nervous system than in the immune system, in some cases 10 to 50 times more so, ${ }^{10}$ hence the term "neurophilins." Interest regarding mechanisms of drug toxicity as well as the normal function of neurophilins in the nervous system is growing.

Immunophilins are classified into two families: cyclophilins and FK506-binding proteins (FKBPs), ubiquitous proteins that share many functions. ${ }^{6,10}$ Within each family, multiple isoforms have differential tissue expression and distinct subcellular localization. Cyclophilin A and FKBP-12 are the bestdescribed members, although more then 30 cyclophilins and more than 20 FKBPs are reported. Immunophilins are well conserved throughout evolution, suggesting that they mediate critical cellular activities. One role is to mediate proper folding of proteins by virtue of prolyl isomerase (rotamase) activity, which is inhibited upon drug binding. Cyclosporin A inhibits cyclophilin rotamase activity, whereas FK506 and rapamycin inhibit rotamase activity of the FKBPs. Although only modest evidence supports a physiological role for immunophilin

Correspondence to: E.L. Feldman

CCC 0148-639X/99/101337-04

(C) 1999 John Wiley \& Sons, Inc. regulation of protein folding, rotamase activity might play roles in such processes as protein trafficking, collagen assembly, and chaperone transport of otherwise insoluble polypeptides. Immunosuppressive drug toxicity might be attributed in part to inhibition of rotamase activity, but drug interactions with other substrates might also result in toxicity. One such substrate is calcineurin, which interacts with CsA and FK506 but not with rapamycin.

Immunophilin-mediated immunosuppression does not involve inhibition of protein folding but rather inhibition of calcineurin, a calcium and calmodulin-dependent serine/threonine protein phosphatase. Calcineurin affects gene expression in response to calcium flux and is necessary for the synthesis of several cytokines. Normal activation of the T-cell receptor leads to elevation of intracellular calcium levels, thereby activating calcineurin. Calcineurin in turn dephosphorylates a variety of proteins including transcription factors, leading to increased interleukin-2 levels and T-cell activation. By blocking these events, calcineurin inhibition mediates immunosuppression. Calcineurin comprises over $1 \%$ of the total brain protein and is widely distributed, with highest concentrations in the hippocampus and corpus striatum, suggesting that immunophilins play a role in neural function. Cyclophilin and FKBP expression overlaps, but certain regions express one or more neurophilins preferentially. It is therefore not surprising that CsA and FK506 have physiological effects in the nervous system, including wellcharacterized central nervous system (CNS) toxicities. Central nervous system localization of the cyclophilins and FKBPs generally parallels that of calcineurin, and CNS expression of all three is much greater than in the immune system. Low concentrations of CsA and FK506 enhance the phosphorylation of endogenous protein substrates in brain tissue and PC12 cells by interacting with calcineurinneurophilin complexes. Important targets of calcineurin in brain are the growth-associated protein GAP-43, N-methyl-D-aspartate (NMDA) receptor 
channels, nitric oxide synthase (NOS), and dynamin I. Calcineurin may thus regulate a number of physiological responses in the CNS, such as neurite extension, transmitter release, long-term potentiation, long-term depression, synaptic vesicle processing, and synaptic plasticity.

Immunophilin ligands are neuroprotective in numerous experimental models. Inhibition of calcineurin by FK506 or CsA is neuroprotective in ischemic, glutamate-mediated excitotoxic and $\beta$-amyloid peptide-mediated models of neurotoxicity and following serum deprivation. ${ }^{3,10}$ Calcineurin also protects striatal neurons from cell death after treatment with MPTP or 6-hydroxydopamine. One possible mechanism of neuroprotection by these drugs is interference with NMDA receptors and NOS activity. However, these drugs have no neuroprotective effect on quisqualate- and kainate-mediated neurotoxicity, suggesting that neuroprotective effects of drugneurophilin complexes involve mechanisms distinct from NMDA-mediated signaling pathways. It should also be noted that CsA (but not FK506 or rapamycin) inhibits the opening of mitochondrial permeability transition pores and may therefore, in some cases, inhibit apoptosis.

Early reports indicated that FK506 may be somewhat less toxic than CsA, though estimates vary widely. Toxicity is due at least in part to calcineurin inhibition. Possible mechanisms of CsA neurotoxicity include microvascular injury with associated reversible ischemia versus direct toxic effects, such as drug-induced apoptosis. ${ }^{7}$ Limited pathological studies of brain have revealed myelin pallor, hemorrhagic foci, neuronal loss (notably in the occipital lobes), and astrocytosis of white matter. It is postulated that calcineurin may regulate normal CNS myelination, and immunophilin-mediated drug inhibition of calcineurin might lead to demyelination. Mechanisms of toxicity in the peripheral nervous system (PNS) remain speculative. To our knowledge, there have been no pathological studies of nerve and only limited studies of muscle.

Initially, it was thought that the PNS and muscle were relatively spared from significant FK506 or CsA toxicity. However, it is now clear that both drugs can cause myopathy and neuropathy. Treatment with either CsA or FK506 can cause a hypertrophic cardiomyopathy or skeletal myopathy. The myopathy is generally mild, with myalgia and muscular weakness but normal creatine kinase levels. In many cases, myopathic symptoms improve with cessation of CsA and recur when the drug is reintroduced. Onset of myopathy is at least several months after the onset of CsA therapy. Pathological findings associated with
CsA-induced myopathy include type 2 fiber atrophy, vacuole formation, segmental necrosis, and occasionally mitochondrial abnormalities. Some of the patients described with this form of myopathy were also taking colchicine concomitantly, which is both myotoxic and neurotoxic. A more severe myopathy with rhabdomyolysis has also occurred in patients taking CsA; all patients were taking a second myotoxic drug in addition to CsA, either lovastatin or colchicine, making it difficult to attribute the myopathy to CsA alone. Nonetheless, it appears from this small number of case reports that CsA is myotoxic, especially when used in combination with other myotoxic drugs.

Treatment with cholesterol-lowering drugs can result in myopathy, as occurs in $0.1 \%$ to $0.2 \%$ of cases of lovastatin monotherapy. Incidence of myopathy increases significantly (possibly as high as 30\%) with coadministration of CsA and lovastatin, perhaps because CsA inhibits the biliary elimination of lovastatin, leading to increased drug levels and myotoxicity. ${ }^{9}$ The mechanism of cell injury, however, and the reason that muscle should be selectively affected, are not understood. Given that muscle activity is critically dependent on calcium flux, we speculate that myotoxicity from CsA and FK506 results from calcineurin inhibition, interfering with calciumregulated pathways. This hypothesis is supported by the finding that calcineurin controls muscle fibertype-specific gene expression, with calcineurin activation upregulating slow fiber-specific gene promoters and calcineurin inhibition (by CsA) promoting slow-to-fast fiber transformation. ${ }^{2}$

A theoretical explanation for the synergistic myotoxicity of CsA and lovastatin is that these drugs interfere with distinct aspects of cholesterol metabolism. In vitro studies show that CsA and rapamycin inhibit cholesterol transport in fibroblasts by disrupting cholesterol-immunophilin-caveolin complexes. ${ }^{11}$ It is not known whether cholesterol transport in neural cells is mediated by neurophilins, although it is tempting to speculate that this might be a mechanism of neurotoxicity. An inhibitor of cholesterol synthesis plus a drug that blocks cholesterol efflux might cause synergistic toxicity by interfering with muscle cell cholesterol metabolism or signal transduction processes. The finding that hypocholesterolemia can significantly increase the risk of developing CsA toxicity supports this hypothesis, but further research is necessary to address this issue.

Several patients have been reported with a severe progressive multifocal sensorimotor neuropathy due to FK506 that resembles chronic inflammatory demyelinating polyradiculoneuropathy (CIDP), except 
that prominent sensory symptoms are noted. ${ }^{12}$ Onset of neuropathy is 2-10 weeks after the first exposure to FK506, with first symptoms typically consisting of paresthesias or painful dysesthesias in the feet. Patients have significant asymmetric weakness. Electrophysiological studies show mainly demyelinating neuropathy, and spinal fluid protein is elevated with normal cell counts. Some improvement follows treatment with plasmapheresis or intravenous immunoglobulin. Among liver transplant patients treated with CsA postoperatively, approximately $6.5 \%$ have isolated peripheral neuropathies and normal spinal fluid studies, and nerve conduction studies suggest underlying demyelination. ${ }^{5}$ High CsA levels have been documented, and recovery occurs within 2 months after lowering drug dosage. Other isolated case reports also ascribe entrapment neuropathies and demyelinating brachial neuropathy to CsA toxicity; some cases are consistent with CIDP, although cerebrospinal fluid (CSF) analysis is often normal.

Cyclosporin A or FK506 may trigger immunologically mediated neuropathies. That is, a dysimmune neuropathy might result from altered T-cell activation or T-cell subset ratios. Changes in T-cell subsets are observed in rats given FK506 or CsA prophylactically to prevent development of experimental autoimmune neuritis (EAN), whereas a relapsing form of EAN is induced by low-dose CsA. ${ }^{1}$ Both CsA and FK506 interfere with intrathymic T-cell development by blocking stimulus-driven apoptosis, and there is some evidence that an increased percentage of thymic T-cells react to self class II multiple histocompatibility antigens in CsA-treated mice, possibly reflecting inhibition of apoptosis by CsA. An alternative mechanism for the observed neuropathic effects of these drugs is direct demyelination. Elemental tellurium causes a well-established experimental demyelinating neuropathy in young rats by blocking cholesterol synthesis, and it is possible that the transport of cholesterol, which makes up a considerable fraction of peripheral myelin, is disrupted by CsA or FK506 in the same way as in vitro. We have recently characterized Schwann cell expression of caveolin- $1^{8}$ and are investigating whether Schwann cell cholesterol transport is mediated by neurophilins.

Immunophilins may function as adapter proteins to couple macromolecular complexes, including the ryanodine receptor (RyR) and the 1,4,5-triphosphate receptor $\left(\mathrm{IP}_{3} \mathrm{R}\right)$, both of which are intracellular calcium channels, and the transforming growth factor (TGF) $\beta 1-\mathrm{R}$. The RyR is localized to the sarcoplasmic reticulum of muscle but is also found in cardiac muscle and brain. It is the calcium release channel involved in excitation-contraction coupling in muscle. FK506 can dissociate FKBP-12 from the RyR, impairing the calcium-fluxing properties of this channel. The $\mathrm{IP}_{3} \mathrm{R}$ is also a calcium release channel found primarily in the endoplasmic reticulum, and because it too binds FKBP-12, its function can be disrupted by FK506 or rapamycin. Calcineurin is associated with both the FKBP-12/RyR and FKBP-12/ $\mathrm{IP}_{3} \mathrm{R}$ complexes, probably through binding to FKBP-12 as a docking molecule. FK506 binding protein-12 also interacts with the TGF $\beta 1-R$, which has serine/threonine kinase activity. In this setting, FKBP-12 may function as an inhibitor of TGF $\beta$-mediated signaling, possibly by acting as a docking molecule for calcineurin.

Neurophilins likely play roles during neural development, disease, and repair. For example, FKBP12 , which is an important mediator of FK506's immunosuppressant activity, is highly expressed in peripheral nerves. Its presence in growth cones of developing neurons suggests a role in nerve growth. Along with GAP-43 induction, FKBP-12 expression increases in facial motor neurons and lumbar nuclei after axotomy, and low-dose FK506 increases the rate of axonal regeneration and functional recovery after sciatic nerve crush injury in a dose-dependent fashion. ${ }^{4}$ Other FKBP-12 inhibitors which have no effect on calcineurin also increase the rate of axonal regeneration, and while some controversy exists, equivalent doses of CsA appear to have no effect, suggesting that calcineurin may not be involved. Higher FK506 doses actually lead to calcineurin inhibition, increased GAP-43 phosphorylation, and impaired regeneration. Low-dose (1 nM or less) FK506 enhances neurite outgrowth of PC12 cells and SHSY5Y human neuroblastoma cells (when nerve growth factor [NGF] is present at low submaximal doses) and dorsal root ganglion explant cultures, whereas CsA does not clearly affect neurite outgrowth, and higher dose FK506 $(50 \mu \mathrm{M})$ actually reduces it.

The target(s) of calcineurin-independent FKBP12 inhibition that is responsible for axonal regeneration or neurite outgrowth is unknown. One candidate is the $\mathrm{IP}_{3} \mathrm{R}$, as inhibition of this receptor reduces neurite outgrowth. FK506 can stabilize the $\mathrm{IP}_{3} \mathrm{R}$, enhancing its receptor activity and possibly accounting for the observed increased neurite outgrowth. Another potential mechanism implicated in FK506's effects on nerve regeneration involves the TGF $\beta 1-R$. FK506 binding protein-12 interacts with the TGF $\beta 1-R$ and inhibits its function. Because TGF $\beta 1$ enhances nerve growth factor (NGF) synthesis by glial cells, FK506 could increase axonal regen- 
eration through increased NGF generation. Transforming growth factor- $\beta$ also induces long-term synaptic facilitation, protects hippocampal neurons from ischemic injury, and promotes axonal regeneration. Further work is necessary to identify the mechanisms by which neurophilin ligands produce their many neural effects, but they likely act downstream of multiple receptors, including neurotrophic receptors.

In summary, CsA, FK506, and rapamycin are a group of immunosuppressant drugs which mediate their effects after binding to various immunophilins and, in the nervous system, to neurophilins. Neurophilins may play roles in neural cell death, neurite outgrowth, synaptic function, and myelination. It is important to recognize the toxic effects of these drugs, because they are generally reversible upon discontinuing or lowering the dose of drug. Neurotoxic effects are often mediated by calcineurin inhibition. As a result, calcium-regulated pathways are dysregulated, which may explain, for example, druginduced myopathy. Toxicity may also be due to rotamase activity or neurophilin-mediated effects on a downstream signaling pathway. It is possible that nervous system-specific neurophilin isoforms exist, which may explain specificity of action. We speculate that neurophilins may regulate Schwann cell cholesterol transport and that interference with this mechanism could lead to PNS toxicity by causing demyelination. Based upon these hypothetical mechanisms of neurophilin action, it may be possible to generate orally active nonimmunosuppressant ligands for FKBPs and/or cyclophilins that could be used in the treatment of a variety of neu- rological disorders such as diabetic neuropathy, amyotrophic lateral sclerosis, stroke, and neurodegenerative disorders such as Alzheimer's disease and Parkinson's disease.

\section{REFERENCES}

1. Adachi A, Araga S, Takahashi K. Immunosuppressive effect of FK506 on experimental allergic neuritis in Lewis rats: change of T cell subsets. Intern Med 1992;31:6-10.

2. Chin ER, Olson EN, Richardson JA, Yang Q, Humphries C, Shelton JM, Wu H, Zhu W, Bassel-Duby R, Williams RS. A calcineurin-dependent transcriptional pathway controls skeletal muscle fiber type. Genes Dev 1998;12:2499-2509.

3. Dawson TM, Steiner JP, Dawson VL, Dinerman JL, Uhl GR, Snyder SH. Immunosuppressant FK506 enhances phosphorylation of nitric oxide synthase and protects against glutamate neurotoxicity. Proc Natl Acad Sci USA 1993;90:9808-9812.

4. Gold BG. FK506 and the role of immunophilins in nerve regeneration. Mol Neurobiol 1997;15:285-306.

5. Guarino M, Stracciari A, Pazzaglia P, Sterzi R, Santilli I, Donato F, D'Alessandro R. Neurological complications of liver transplantation. J Neurol 1996;243:137-142.

6. Hamilton GS, Steiner JP. Immunophilins: beyond immunosuppression. J Med Chem 1998;41:5119-5143.

7. McDonald JW, Goldberg MP, Gwag BJ, Chi SI, Choi DW. Cyclosporine induces neuronal apoptosis and selective oligodendrocyte death in cortical cultures. Ann Neurol 1996;40: $750-758$.

8. Mikol DD, Hong H, Cheng H-L, Feldman EL. Caveolin-1 expression in Schwann cells. Glia 1999;27(1)39-52.

9. Smith PF, Eydelloth RS, Grossman SJ, Stubbs RJ, Schwartz MS, Germershausen JI, MacDonald JS. Myopathy associated with HMG-CoA reductase inhibitors (HMGRIs) and cyclosporin A: evaluation in a rat model. Eur Heart J 1992;13 Suppl B:2-6.

10. Snyder SH, Lai MM, Burnett PE. Immunophilins in the nervous system. Neuron 1998;21:283-294.

11. Uittenbogaard A, Ying Y, Smart EJ. Characterization of a cytosolic heat-shock protein-caveolin chaperone complex. J Biol Chem 1998;273:6525-6532.

12. Wilson JR, Conwit RA, Eidelman BH, Starzl T, Abu-Elmagd K. Sensorimotor neuropathy resembling CIDP in patients receiving FK506. Muscle Nerve 1994;17:528-532. 